\title{
Loi de Poiseuille dans une conduite cylindrique obtenue par aplatissement uniforme d'un tube initialement elliptique
}

\author{
A. Langlet - S. Naili - C. Ribreau \\ Laboratoire de Mécanique Physique, U.F.R. de Sciences et Technologie, \\ Université Paris 12-Val de Marne, Avenue du Général-de-Gaulle, F 94010 Créteil Cedex
}

\section{Introduction}

Même si quelques hydrauliciens, comme par exemple VISCHER et coll. [13], s'aventurent parfois sur le terrain des tuyaux collabables, même si quelques chercheurs peuvent aller dans un grand élan de transfert technologique jusqu'à concevoir un composant hydraulique de régulation (RODBARD [11]), voire une expérience dite d'amateur ( - arrosez rapidement votre jardin... en bouchant le tuyau (WALKER [14]) -), les applications des écoulements en conduite collabable relèvent moins de l'hydraulique traditionnelle que de la biomécanique, qu'on en juge d'après ces quelques exemples : « How giraffes prevent oedema (PEDLEY [8])», « External compression as prophylaxis against deep vein thrombosis (KAMM [6]) ", " Hydrodynamics of male micturation (GRIFFITHS [4]) ", "A model of the vocal cords (CONRAD [2]) », " Hémodynamique veineuse (RIBREAU [9]) ", "Flow limitation during forced expiration (THIRIET et coll. [12]) ". La théorie des écoulements en conduite collabable relève de la mécanique des systèmes non linéaires de milieux continus, fluide-paroi, couplés.

\begin{abstract}
Sa mise en œuvre la plus simple requiert d'une part, une formulation du changement d'état de la conduite et d'autre part, une formulation de la perte de charge locale.

Dans une conduite cylindrique, c'est-à-dire de section constante, le coefficient de perte de charge $\Lambda=f / R_{e}$ de l'écoulement laminaire est défini à partir du facteur $f$, dit coefficient de forme, spécifique de la géométrie de la section, et du nombre de Reynolds $R_{e}$. Nous proposons de déterminer les coefficients de forme dans différentes conduites cylindriques dont les sections sont définies avec la loi de changement d'état d'un tube initialement elliptique comprimé uniformément. La loi de changement d'état présente quatre sections caractéristiques: $S_{0}, S_{p}, S_{c}, S_{1}$. La méthode empirique proposée permet à partir des coefficients $f_{0}, f_{p}, f_{1}$, associés aux sections $S_{0}, S_{p}, S_{1}$, d'accéder, par extrapolation, au coefficient de forme d'une section quelconque.

La connaissance de l'évolution du coefficient de forme en fonction de la géométrie des sections écrasées permet donc de traiter les équations différentielles de l'écoulement unidimensionnel permanent dans une conduite collabable.
\end{abstract}

\section{Poiseuille's law in cylindrical ducts obtained by flattening of elliptical tubes}

Elastic tubes can collapse if the transmural pressure (internal pressure minus external pressure) is negative. A small viscous or inertial pressure drop accompanying fluid through the tube may be enough to cause a large reduction in area. Such coupling of flow through an elastic tube with collapse of the tube arises in veins or self-controlled flow rate and pressure regulator devices. The one-dimensional models of flow through these collapsible tubes involve the knowledge of regular head loss in cylindrical pipes with buckled cross-sections. In this paper we determine the head loss in laminar flow for such pipes. 


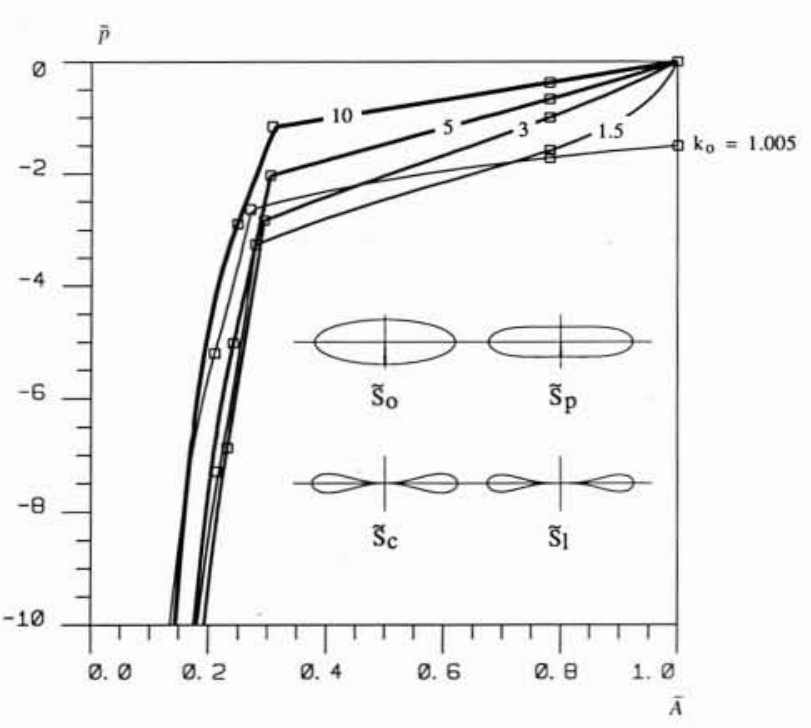

1. Loi de changement d'état (pression adimensionnée/aire normalisée) d'un tube mince elliptique pour différentes valeurs de l'ellipticité $k_{0}$.

Dimensionless theoretical pressure/area law of elliptical thinwalled tubes for various values of the ellipticity $k_{0}$.

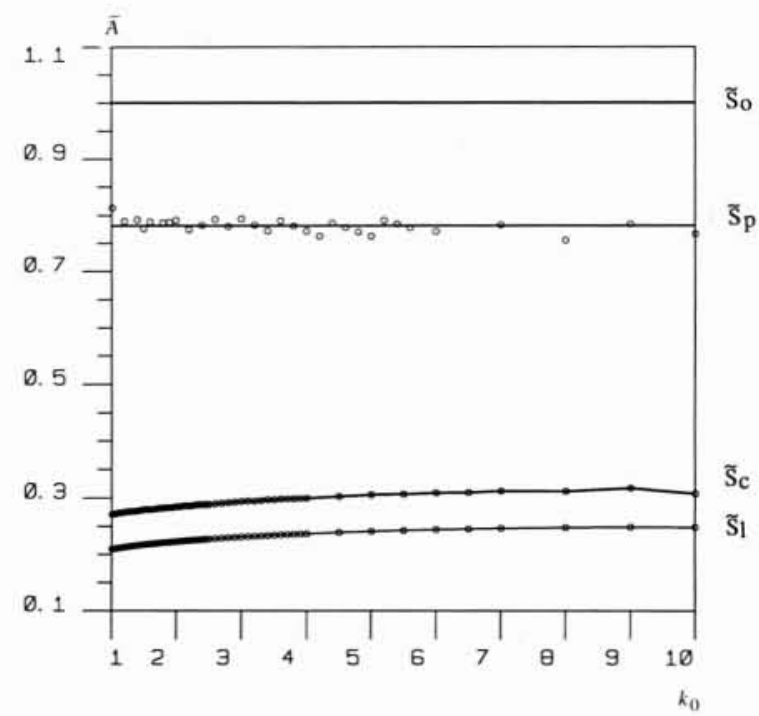

2. Evolution des quatre aires normalisées de quatre sections caractéristiques $\tilde{S}_{0}, \hat{S}_{p}, \tilde{S}_{c}, \tilde{S}_{1}$ d'un tube mince elliptique comprimé uniformément en fonction de l'ellipticité $k_{0}$. $A / A_{0}$ against $k_{0}$ for the particular shapes $\tilde{S}_{0}, \tilde{S}_{p}, \tilde{S}_{c}$, $\hat{S}_{1}$ of an elliptical thin-walled tubes during collapse.

\section{Loi de changement d'état}

\subsection{Description}

Nous utilisons les résultats de BonIs et coll. [1] qui portent sur la déformation d'un tube mince élastique cylindrique homogène uniforme de longueur infinie soumis à une compression uniforme. La section elliptique initiale $S_{0}$ est particularisée par le rapport $k_{0}$ (ellipticité) du demi grand axe $a_{0}$ au demi petit axe $b_{0}$. La déformation s'effectue à périmètre $\chi_{0}$ constant.

Les pressions transmurales $p$ (pression intérieure $p_{i}$ moins pression extérieure $p_{e}$ ), les aires $A$ des sections $S$, les longueurs relatives à la section, comme par exemple les coordonnées ( $y$ et $z$ ) d'un point du contour et le périmètre, sont adimensionnées avec le coefficient $K=E H_{0}^{3}\left[6\left(1-v^{2}\right) a_{0}^{3}\right]$ et le demi grand axe $a_{0}$ de l'ellipse initiale, de sorte que :

$$
\begin{gathered}
\tilde{p}=\frac{p}{K}, \\
\tilde{A}=\frac{A}{a_{0}^{2},} \\
\tilde{y}=\frac{y}{a_{0}}, \quad \tilde{z}=\frac{z}{a_{0}}, \quad \tilde{\chi}_{0}=\frac{\chi_{0}}{a_{0}},
\end{gathered}
$$

$S$ devienne $\tilde{S}$.
Le module d'Young, le coefficient de Poisson, l'épaisseur de la paroi, ont été notés $E$, v et $H_{0}$.

En fonction de la pression transmurale $\tilde{p}$, ou de l'aire normalisée $\bar{A}=\tilde{A} / \tilde{A}_{0}$, la section droite peut se placer dans un des quatre cas suivants :

a) $\tilde{p}=0, \bar{A}_{0}=1$, la section est elliptique (section $\left.\tilde{S}_{0}\right)$.

b) $\tilde{p}=\tilde{p}_{p}, \bar{A}=\bar{A}_{p}$, les deux faces opposées de la paroi sont quasiment planes (section $\tilde{S}_{p}$ ). La section $\tilde{S}_{p}$ est caractérisée par le rapport $k_{p}=a_{p} / b_{p}$ de son grand axe à son petit axe.

c) $\tilde{p}=\tilde{p}_{c}$ pression de contact, $\bar{A}=\bar{A}_{c}$, la section possède un point de contact (section $\tilde{S}_{c}$ ). La section $\tilde{S}_{c}$ correspond à un point anguleux de la fonction $\tilde{p}(\bar{A})$.

d) $\tilde{p}=\tilde{p}_{l}$ pression de ligne, $\bar{A}=\bar{A}_{l}$, le rayon de courbure au point de contact tend vers l'infini, la section présente toujours un point de contact (section $\tilde{S}_{1}$ ). Pour les valeurs inférieures à la pression de ligne, un segment de contact rectiligne se développe.

La fonction $\tilde{p}(\bar{A})$ est représentée dans la figure 1 pour cinq valeurs de $k_{0}$ (1,005-1,5-3-5-10) et l'évolution des aires des quatre sections caractéristiques est montrée dans la figure 2 : quelle que soit la valeur de $k_{0}$ comprise entre 1 et 10 , l'aire $\bar{A}_{p}$ des sections $\tilde{S}_{p}$ est quasiment constante (voisine de 0,78 ). 


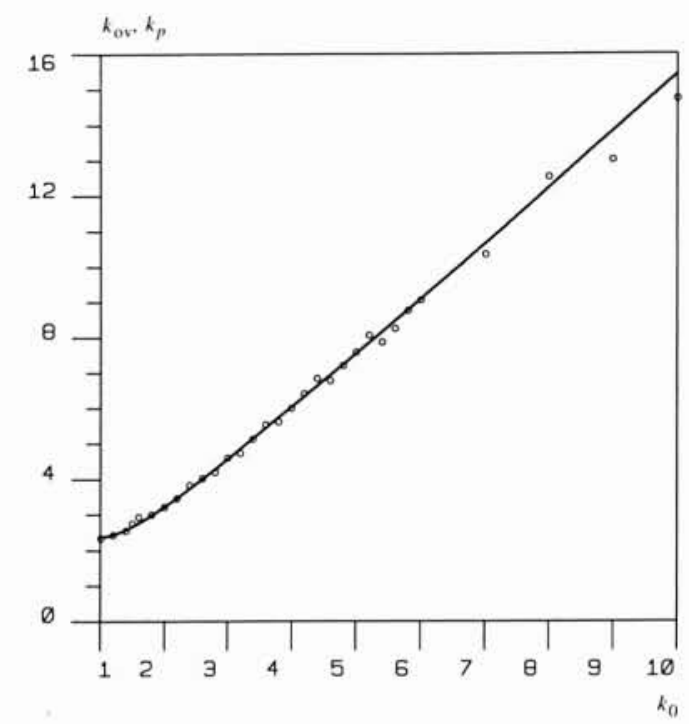

3. Rapport $k_{\text {ov }}$ (ligne continue) du demi grand axe par le demi petit axe de la section ovale $\bar{S}_{\text {ov }}$ approchant la section $\tilde{S}_{p}$ et rapport $k_{p}$ (symboles circulaires) du demi grand axe par le demi petit axe de la section $\tilde{S}_{p}$ en fonction de l'ellipticité $k_{0}$. L'ovale et la section $\bar{S}_{p}$ ont même aire et même périmètre.

Major to minor axis ratio $k_{o v}$ (continuous line) from the cross-sectional shape $\tilde{S}_{p}$ approximated as an oval $\tilde{S}_{w}$ and major to minor axis ratio $k_{p}$ (circle symbols) from the numerical cross-sectional shape $\tilde{S}_{p}$ against $k_{0}$. The sections $\tilde{S}_{\mathrm{ov}}$ and $\tilde{S}_{p}$ have the same area and the same perimeter.

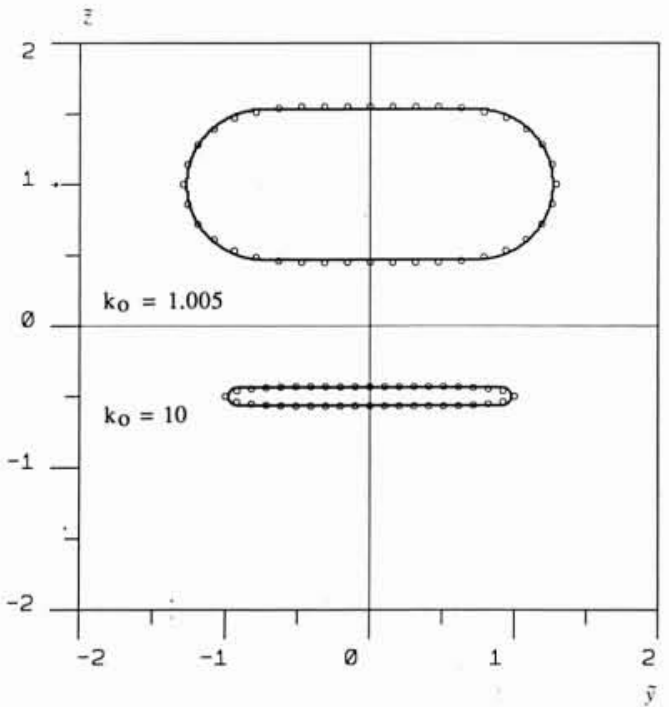

4. Comparaison entre la forme de la section $\bar{S}_{p}$ (symboles circulaires) et la forme ovale (ligne continue) pour les valeurs 1,005 et 10 de $k_{0}$.

The numerical cross-sectional shape $\tilde{S}_{p}$ (circle symbols) compared with the oval shape (continuous line) for the values 1.005 and 10 of $k_{0}$.

\subsection{Approximation de la section $\tilde{S}_{p}$ par une section ovale}

L'ovale qui approche la section $\tilde{S}_{p}$ est un rectangle bordé par deux demi cercles de rayon $b_{\text {ov }}$. Le rapport des demiaxes s'écrit

$$
k_{\mathrm{ov}}=\frac{a_{\mathrm{ov}}}{b_{\mathrm{ov}}} .
$$

Le périmètre $\tilde{\chi}_{\mathrm{ov}}$ et l'aire $\bar{A}_{\mathrm{ov}}$ de l'ovale correspondent aux valeurs constantes $\tilde{\chi}_{0}$ et $\bar{A}_{p}$, il s'ensuit la relation entre $k_{\text {ov }}$ et $k_{0}$ tracée figure 3 conjointement aux valeurs $k_{p}\left(k_{0}\right)$. L'adéquation entre les deux types de résultats est correcte jusqu'à $k_{0}=8$, au-delà l'approximation par deux demi-cercles est plus grossière, voir figure 4 .

\subsection{Solutions analytiques pour $\tilde{\boldsymbol{p}}<\tilde{\boldsymbol{p}}_{i}$}

Lorsque $k_{0}$ vaut 1 , il a été montré par FLAHERTY et coll. [3] une solution de similitude entre l'état mécanique de référence, défini à la pression $\tilde{p}_{1}$, et un état quelconque tel que $\tilde{p}<\tilde{p}_{1}$. Pour une valeur quelconque de $k_{0}$ et $\tilde{p} / \tilde{p}_{1} \leqslant 10$, cette propriété peut être étendue. Une solution approchée d'analogie, mais non de similitude, est démontrée (RIBREAU [10]). Les fonctions analytiques $G_{11}, G_{1}, G_{2}, G_{3}, G_{4}$ décrivant respectivement l'aire de la section, le périmètre, la distorsion angulaire et les coordonnées $(\tilde{y}, \tilde{z})$ s'écrivent :

$$
\begin{gathered}
G_{11}=\left(\bar{A} / \bar{A}_{1}\right)=\left(\tilde{p} / \tilde{p}_{1}\right)^{-1 / n}, \\
G_{1}=\left(\tilde{x} / \tilde{x}_{1}\right)=\left(G_{11} /\left(\tilde{p} / \tilde{p}_{1}\right)\right)^{1 / 5}, \\
G_{2}=\left(\tilde{\theta} / \tilde{\theta}_{1}\right)=\left(G_{11}^{3}\left(\tilde{p} / \tilde{p}_{1}\right)^{2}\right)^{1 / 5}, \\
G_{3}=\left(\tilde{y} / \tilde{y}_{1}\right) \cong G_{1}, \\
G_{4}=\left(\tilde{z} / \tilde{z}_{1}\right) \cong G_{3} G_{2} .
\end{gathered}
$$

L'exposant $n$ est déduit des calculs numériques. Il varie entre $3 / 2$ et $9 / 4$ lorsque $k_{0}$ croît de 1 à 10 . Il est à noter que la valeur $3 / 2$ correspond à la solution de similitude.

\section{Ecoulement laminaire dans une conduite cylindrique. Loi de Poiseuille}

L'aire constante $A$ de la section $S$ est définie à partir de la loi $p(A)$ décrite ci-dessus. Les coordonnées d'un point à l'intérieur du domaine de fluide sont notées $x_{f}$, $y_{f}$ et $z_{f}$. L'écoulement est établi : dans chaque section droite de la conduite la vitesse en un point est notée $\vec{V}\left(y_{f}, z_{f}\right)=u\left(y_{f}, z_{f}\right) \vec{i}$. La projection de l'équation de Navier-Stokes sur l'axe $x$ de la conduite cylindrique de 
longueur $L_{x}$, pour un fluide incompressible de masse volumique $\rho$ et de viscosité dynamique $\mu$, s'écrit alors :

$$
0=-\alpha_{v}+\mu \Delta\left[u\left(y_{f}, z_{f}\right)\right] .
$$

Le gradient de pression de l'écoulement, $\mathrm{d} p_{f} / \mathrm{d} x_{f}=\alpha_{v}$, peut être formulé de deux manières :

a) Il est proportionnel au débit volumique (le facteur de proportionnalité $\sigma$ est appelé conductance)

$$
\alpha_{v}=-\frac{q}{\sigma} .
$$

b) Il est exprimé à partir de la formule de DarcyWeisbach dans laquelle $\Lambda=f / R_{e}$, (le périmètre mouillé de la section de conduite est noté $\chi$ )

$$
\alpha_{v}=-\Lambda \chi \frac{\rho q^{2}}{8 A^{3}}=-\mu \frac{f \chi^{2} q}{32 A^{3}} .
$$

Utilisant le jeu de variables sans dimension suivant :

$$
\begin{gathered}
\tilde{x}_{f}=\frac{x_{f}}{L_{x}} ; \quad \tilde{y}_{f}=\frac{y_{f}}{a_{0}} ; \quad \tilde{z}_{f}=\frac{z_{f}}{a_{0}} ; \\
\tilde{p}_{f}=\frac{p_{f}}{K} ; \quad \bar{A}=\frac{\tilde{A}}{\tilde{A}_{0}} ; \quad \bar{u}=-\frac{\mu}{\alpha_{v}} \frac{u}{a_{0}^{2}} ;
\end{gathered}
$$

il vient :

$\Delta\left[\bar{u}\left(\tilde{y}_{f}, \tilde{z}_{f}\right)\right]=-1$ avec $\bar{u}=0$ sur le bord de la section $\tilde{S}$,

$$
\begin{gathered}
\tilde{\boldsymbol{\sigma}}=\frac{\mu \sigma}{a_{0}^{4}}=\int_{\tilde{s}} \bar{u} \mathrm{~d} \tilde{y}_{f} \mathrm{~d} \tilde{z}_{f}, \\
\frac{f}{f_{0}}=\frac{\tilde{\boldsymbol{\sigma}}_{0}}{\tilde{\sigma}}\left(\frac{\tilde{x}_{0}}{\tilde{\chi}}\right)^{2} \bar{A}^{3} .
\end{gathered}
$$

L'indice « 0 » repère les variables relatives à la section initiale $\tilde{S}_{0}$.

Les traitements numériques portant successivement sur $\bar{u}$ et la conductance $\tilde{\sigma}$ présentent l'inconvénient d'être longs et fastidieux : il faut en effet déterminer le contour de toutes les sections puis les mailler une par une (M.E.F.), ellipticité par ellipticité. Pour traiter la généralité de ce problème, et rendre les résultats directement exploitables, nous avons choisi une méthode de résolution (de compromis) combinant calcul numérique et calculs analytiques. Les calculs analytiques s'appliquent à $f_{0}\left(k_{0}\right), f_{p}\left(k_{0}\right)$ et, quand $\tilde{p}<\tilde{p}_{1}$, à $f\left(k_{0}\right)$. Le calcul numérique s'applique à $f_{1}\left(k_{0}\right)$.

Dans cette présentation nous avons supposé que la fonction $f(\bar{A}) / f_{0}$ est une fonction affine sur les intervalles $\left[\bar{A}_{p}-1\right]$ et $\left[\bar{A}_{1}-\bar{A}_{p}\right]$ et qu'elle atteint sa valeur maximale pour la section $\tilde{S}_{p}$.

\section{Calcul des coefficients de forme}

\subsection{Coefficient $f_{0}$ de I'ellipse $\tilde{S}_{0}$}

La conductance $\sigma_{0}$ et le coefficient de forme $f_{0}$ sont donnés par les formules classiques:

$$
\begin{gathered}
\frac{\mu \sigma_{0}}{a_{0}^{4}}=\frac{\pi}{4} \frac{1}{k_{0}^{3}} \frac{1}{\left(1+\frac{1}{k_{0}^{2}}\right)} \\
f_{0}=128 \pi^{2}\left(1+\frac{1}{k_{0}^{2}}\right) \frac{1}{\tilde{\chi}_{0}^{2}} .
\end{gathered}
$$

La fonction $f_{0}\left(k_{0}\right)$ varie entre 64 et la valeur asymptotique $8 \pi^{2} \approx 79$, figure 5 .

\subsection{Coefficient $f_{p}$ de l'ovale $\tilde{S}_{p}$}

Considérons un rectangle de longueur $2 a_{\mathrm{ov}}$ et de largeur $2 b_{\mathrm{ov}}$ dans lequel s'inscrit une section ovale. D'après les travaux de Hill et Power [5], si le débit dans les deux conduites est le même, la force de frottement visqueux $F_{r}$ par unité de longueur dans la conduite rectangulaire est inférieure ou égale à la force $F_{\text {ov }}$ dans la conduite ovale. Dans le cas de l'écoulement laminaire, sachant que le débit est proportionnel au gradient de pression, on peut écrire entre les conductances $\sigma_{r}$ et $\sigma_{\mathrm{ov}}$ associées respectivement à la conduite rectangulaire et à la conduite ovale :

$$
\frac{A_{r}}{\sigma_{r}} \leqslant \frac{A_{\mathrm{ov}}}{\sigma_{\mathrm{ov}}} .
$$

La discussion de cette relation fait l'objet du travail de ZARLING [15]; il montre que si $k_{\text {ov }}$ est supérieur à environ 2,8 :

$$
\frac{A_{r}}{\sigma_{r}}=\frac{A_{\mathrm{ov}}}{\sigma_{\mathrm{ov}}} .
$$

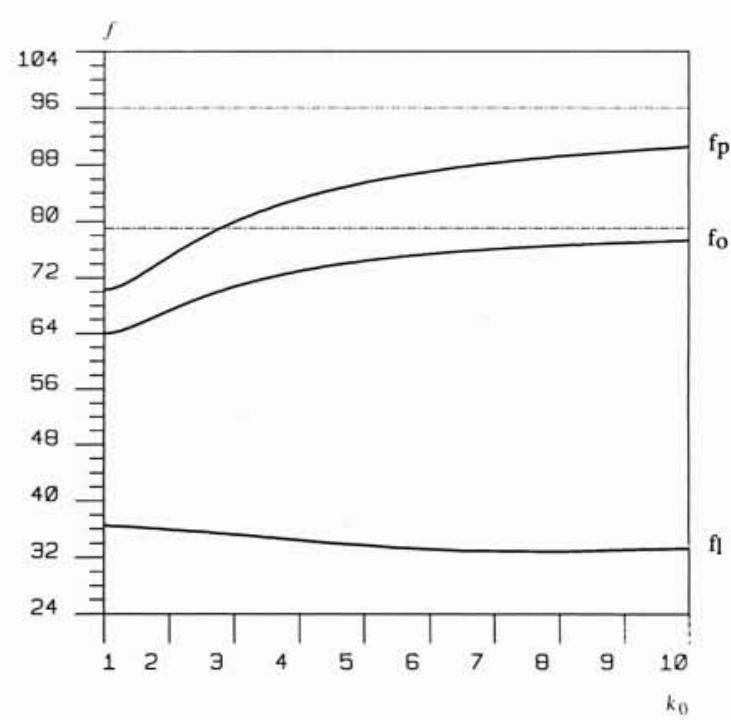

5. Evolution des trois coefficients de forme $f_{0}, f_{p}, f_{1}$, relatifs aux sections $\tilde{S}_{0}, \tilde{S}_{p}, \tilde{S}_{1}$, avec l'ellipticité $k_{0}$. Les valeurs asymptotiques de $f_{0}$ et de $f_{p}$ sont tracées en trait mixte. Characteristical hydraulic shape factors of the three particular sections $\tilde{S}_{0}, \tilde{S}_{p}, \tilde{S}_{1}$, against $k_{0}$. The asymptotic values of $f_{0}$ and $f_{p}$ are shown by the mixed line. 


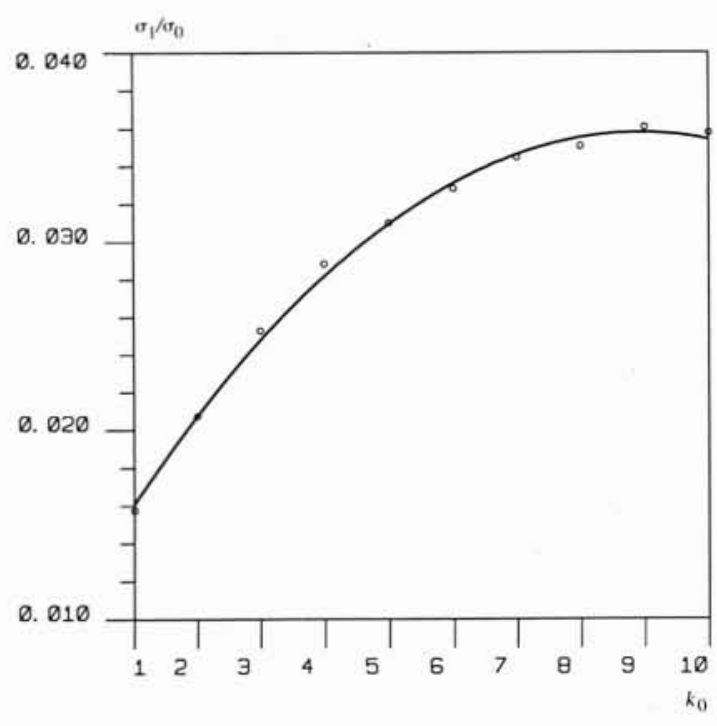

6. Régression quadratique du rapport de la conductance $\sigma_{1}$ par la conductance $\sigma_{0}$ en fonction de $k_{0}$.

Regression curve fit of the $\sigma_{1}$ to $\sigma_{0}$ conductivities ratio against $k_{0}$.

7a. Maillage utilisé pour résoudre $\Delta\left[\bar{u}\left(\tilde{y}_{f}, \tilde{z}_{f}\right)\right]=-1$ dans la section $\tilde{S}_{1}$ correspondant à $k_{0}=1,005$.

An example of mesh grid used for solving $\Delta\left[\bar{u}\left(\tilde{y}_{f}, \tilde{z}_{f}\right)\right]=$ -1 in the particular shape $\bar{S}_{1}$, when $k_{0}=1.005$.

b. Solution présentée sous forme de lignes isovitesses. The solution is shown with isospeed lines.

C. Profil des vitesses sur l'axe longitudinal de symétrie. Velocity profile on the longitudinal y-axis of the section.
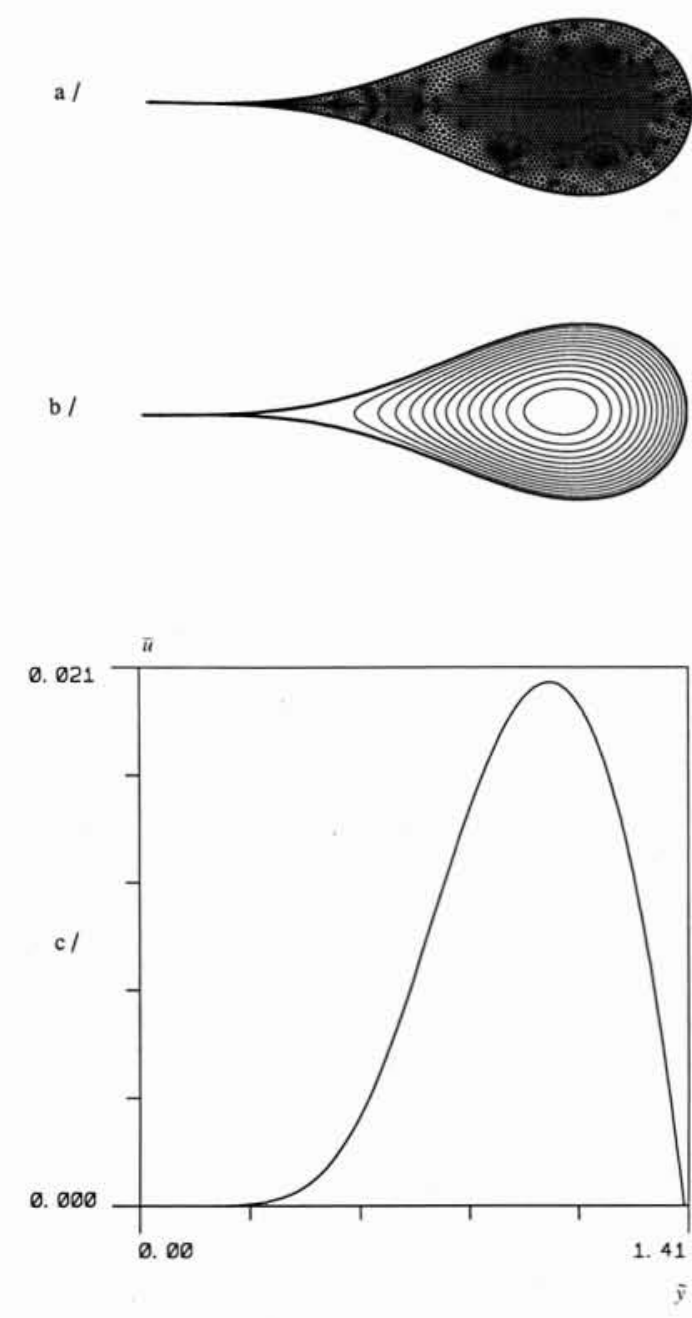

Sachant que la valeur minimale de $k_{\text {ov }}$ est 2,38 pour $k_{0}=1$ et que $k_{\text {ov }}$ augmente rapidement avec $k_{0}$, il est raisonnable d'admettre que pour toutes valeurs de l'ellipticité $k_{0}$ traitées, la conductance de la section ovale est donnée par la relation précédente, soit :

$$
\sigma_{\mathrm{ov}}=\sigma_{r} \frac{4\left(k_{\mathrm{ov}}-1\right)+\pi}{4 k_{\mathrm{ov}}} \text {. }
$$

L'erreur relative sur la conductance $\sigma_{o v}$, évaluée selon ZARLING à $4 \%$, est maximale quand $k_{0}$ vaut 1 .

La conductance $\sigma_{r}$ est donnée par la formule :

$$
\begin{aligned}
\frac{\mu \sigma_{r}}{a_{\mathrm{ov}}^{4}} & =\frac{4}{3 k_{\mathrm{ov}}^{3}} \times \\
& \times\left\{1-\frac{192}{\pi^{5} k_{\mathrm{ov}}} \sum_{i=0}^{\infty} \frac{1}{(2 i+1)^{5}} \text { th }\left[(2 i+1) \frac{\pi k_{\mathrm{ov}}}{2}\right]\right\} .
\end{aligned}
$$

L'expression du coefficient de forme de la section ovale est donc :

$$
f_{p}=96 \frac{\left[\frac{4\left(k_{\mathrm{ov}}-1\right)+\pi}{4\left(k_{\mathrm{ov}}-1\right)+2 \pi}\right]^{2}}{\left\{1-\frac{192}{\pi^{5} k_{\mathrm{ov}}} \sum_{i=0}^{\infty} \frac{1}{(2 i+1)^{5}} \text { th }\left[(2 i+1) \frac{\pi k_{\mathrm{ov}}}{2}\right]\right\}}
$$

La fonction $f_{p}\left(k_{0}\right)$ varie entre 70 et la valeur asymptotique 96 , figure 5 .

\subsection{Coefficient $f_{1}$ de la section $\tilde{S}_{1}$}

Pour calculer $f_{1}$, figure 5 , nous résolvons dans un premier temps l'équation de Poisson par une méthode des éléments finis utilisant un maillage formé de triangles P1. Dans un second temps la solution $\bar{u}\left(\tilde{y}_{f}, \tilde{z}_{f}\right)$ est intégrée numériquement sur toute la section $\tilde{S}_{1}$, ce qui conduit à la valeur de la conductance.

Nous avons traité dix sections $\tilde{S}_{1}$, pour les valeurs $1,005,2,3,4,5,6,7,8,9$ et 10 de $k_{0}$. Les différentes valeurs de $\sigma_{1} / \sigma_{0}$ sont données dans la figure 6 et regroupées autour de la courbe d'équation :

$$
s_{1}\left(k_{0}\right)=\frac{\sigma_{1}}{\sigma_{0}}=0,0108+0,0056 k_{0}-3,13910^{-4} k_{0}^{2} .
$$

Finalement, le coefficient de forme d'une section $\tilde{S}_{1}$ s'écrit :

$$
f_{1}=f_{0} \frac{\bar{A}_{1}^{3}}{\delta_{1}\left(k_{0}\right)} \text {. }
$$

Figure 7, un exemple de maillage et de la solution $\bar{u}\left(\tilde{y}_{f}, \tilde{z}_{f}\right)$, visualisée sous forme de lignes isovaleurs, est présenté pour $k_{0}=1,005$. 


\subsection{Coefficient de forme quand $\tilde{p}<\tilde{p}_{1}$}

Soit une section $\tilde{S}$ définie par $\tilde{p}$ et $k_{0}$. En appliquant la loi d'analogie décrite ci-dessus, on considère, en fait, que la section $\tilde{S}$ est semblable à la section $\tilde{S}_{11}$ qui provient d'une ellipse $\tilde{S}_{00}$ définie par l'ellipticité

$$
k_{00}=\frac{a_{00}}{b_{00}}=\frac{k_{0}}{G_{2}} .
$$

La conductance de la section $\tilde{S}$ vérifie donc :

$$
\frac{\mu \sigma}{G_{1}^{4} a_{00}^{4}}=\frac{\mu \sigma_{11}}{a_{00}^{4}} .
$$

Introduisant le polynôme $S_{1}\left(k_{0}\right)$, on obtient

$$
\frac{\sigma}{\sigma_{1}}=G_{1}{ }_{1} \frac{\sigma_{00}}{\sigma_{0}} \frac{\delta_{1}\left(k_{00}\right)}{S_{1}\left(k_{0}\right)},
$$

et puisque $a_{0}=a_{00}=1$ (valeur de référence des calculs de $\tilde{p}(\bar{A})$ ),

$$
\begin{aligned}
& \frac{\sigma}{\sigma_{1}}=G_{1}^{4} G_{2} \frac{1+k_{0}^{2}}{1+k_{0}^{2} / G_{2}^{2}} \frac{\delta_{1}\left(k_{0} / G_{2}\right)}{\delta_{1}\left(k_{0}\right)}, \\
& \frac{f}{f_{0}}=\frac{\bar{A}^{3}}{G_{1}^{6} G_{2}} \frac{1+k_{0}^{2} / G_{2}^{2}}{1+k_{0}^{2}} \frac{1}{\delta_{1}\left(k_{0} / G_{2}\right)} .
\end{aligned}
$$

\section{Résultats. Discussion}

Nous présentons les tracés des expressions $f(\bar{A}) / f_{0}$ et $f(\tilde{p}) / f_{0}$ dans les figures 8 et 9 pour six valeurs de $k_{0}(1,005-1,5-2-3-5-10)$. Les courbes $f(\tilde{p}) / f_{0}$ sont déduites des courbes $f(\bar{A}) / f_{0}$ grâce à la loi de changement d'état $\bar{A}(\tilde{p})$.

Sur l'intervalle $\left[0-\bar{A}_{1}\right]$, pour une ellipticité donnée $k_{0}$ différente de 1 , les coefficients de forme $f(\bar{A})$, ou le rapport $f(\bar{A}) / f_{0}$, varient peu. Par exemple, si $k_{0}=10$, on trouve $f\left[\bar{A}\left(10 \tilde{p}_{1}\right)\right]=33,99$ et $f\left(\bar{A}_{1}\right)=33,22$. Dans le cas particulier où $k_{0}=1$, la loi de similitude présentée au paragraphe (2.3) conduit à la valeur constante $f_{1}\left(\bar{A}_{1}\right)$.

Par contre, lorsque $\bar{A}=\bar{A}_{1}$ l'effet marqué de $k_{0}$ sur les valeurs de $f(\bar{A}) / f_{0}$ traduit la variation prédominante de $f_{0} \quad$ (fig. 5). On relèvera notamment les valeurs $f\left(\bar{A}_{1}, k_{0}=1,005\right) / f_{0}=0,57 \quad$ et $\quad f\left(\bar{A}_{1}, k_{0}=10\right) /$ $f_{0}=0,43$, soit une diminution de $24 \%$, liée à une augmentation de $21 \%$ de $f_{0}$. D'autre part, les deux fonctions $f_{0}\left(k_{0}\right)$ et $f_{p}\left(k_{0}\right)$ présentant des variations du même ordre, figure 5, aux bornes de l'intervalle $\left[\bar{A}_{p}-1\right]$, il s'ensuit que le rapport $f(\bar{A}) / f_{0}$ dépend faiblement de $k_{0}$ sur cet intervalle. Par suite, pour $\bar{A}$ compris dans l'intervalle $\left[\bar{A}_{1}-\bar{A}_{p}\right]$, la variation de $f(\bar{A}) / f_{0}$ avec $k_{0}$ est fortement influencée par l'ellipticité.

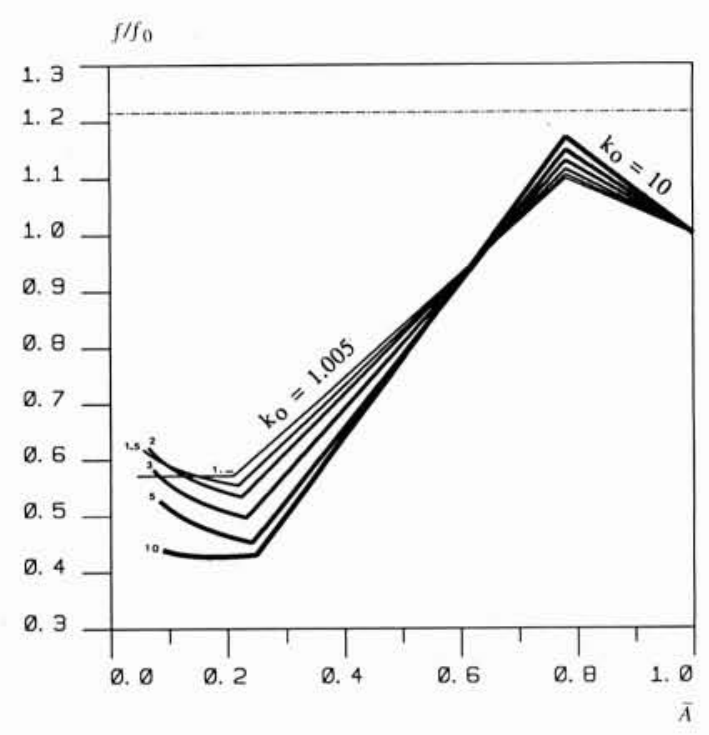

8. Courbe $f(\bar{A}) / f_{0}$ pour six valeurs de $k_{0}$ (1,005-1,5-2-3-5-10). Sur les segments $\left[\bar{A}_{1}-\bar{A}_{p}\right]$ et $\left[\bar{A}_{p}-1\right]$ la fonction $f(\bar{A}) / f_{0}$ est affine. L'aire minimale de $\bar{A}(\tilde{p})$ correspond à $\tilde{p}=$ $10 \tilde{p}_{1}$.

Hydraulic shape factors $f(\bar{A}) / f_{0}$ against the dimensionless area $\bar{A}$ for six values of $k_{0}$ (1.005-1.5-2-3-5-10). Over the intervals $\left[\bar{A}_{1}-\bar{A}_{p}\right]$ and $\left[\bar{A}_{p}-1\right] f(\bar{A}) / f_{0}$ is a linear function. The smaller area $\bar{A}(\tilde{p})$ is for $\tilde{p}=10 \tilde{p}_{1}$.

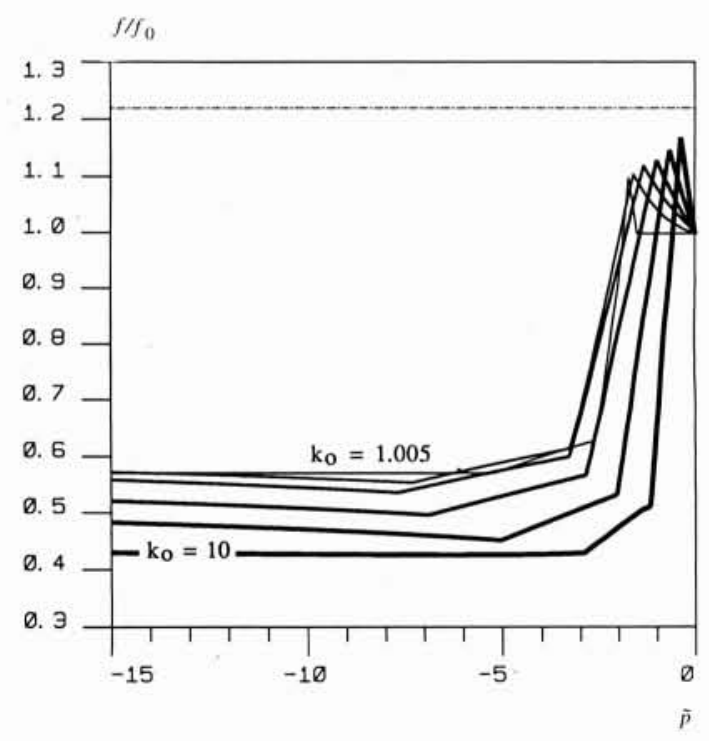

9. Courbe $f(\tilde{p}) / f_{0}$ pour six valeurs de $k_{0}$ (1,005-1,5-2-3-5-10). Hydraulic shape factors $f(\tilde{p}) / f_{0}$ against the dimensionless transmural pressure $\tilde{p}$ for six values of $k_{0}(1.005-1.5-2-3-5$. 10). 


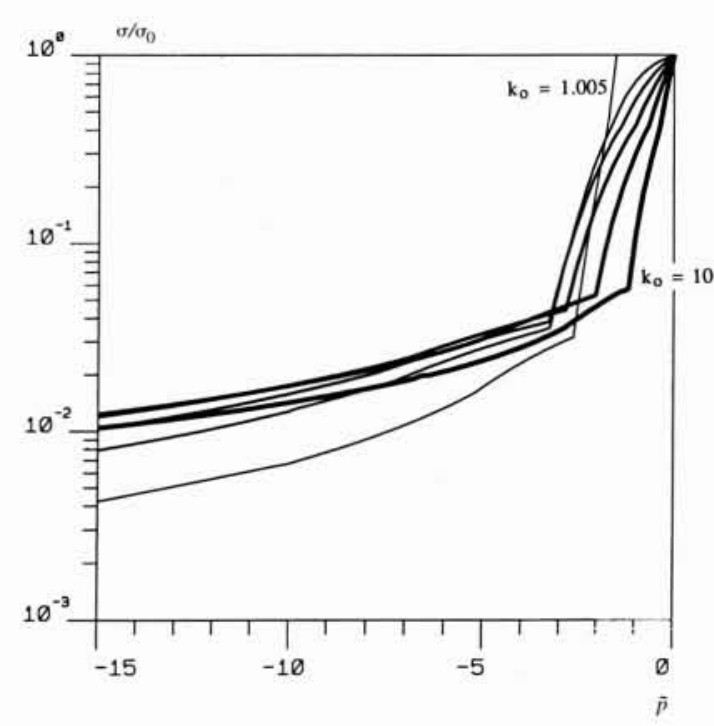

10. Rapport des conductances $\sigma$ par la conductance $\sigma_{0}$ dans des conduites cylindriques en fonction de la pression transmurale normalisée $\tilde{p}$ pour $k_{0}$ valant 1,005-1,5-2-3-510.

The $\sigma$ to $\sigma_{0}$ conductivities ratio of cylindrical buckled tubes as a function of the dimensionless transmural pressure $\tilde{p}$ for the $k_{0}$ values 1.005-1.5-2-3-5-10.

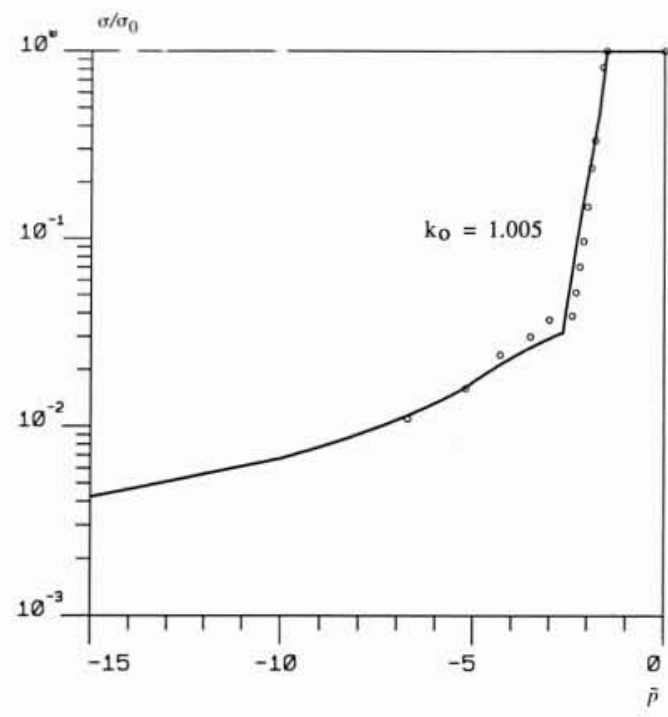

11. Rapport des conductances $\sigma$ par la conductance $\sigma_{0}$ dans des conduites cylindriques issues de la déformation d'un tube initialement circulaire en fonction de la pression transmurale normalisée $\bar{p}$. Comparaison avec quinze valeurs (symboles en forme de cercle) calculées par Flaherty et coll. [3].

The $\sigma$ to $\sigma_{0}$ conductivities ratio of cylindrical buckled tubes, that results from the collapse of an initially circular tube, against the dimensionless transmural pressure $\tilde{p}$. Comparison with fifteen values (circle symbols) of Flaherty's computations [3].
Le réseau des courbes $\sigma(\tilde{p}) / \sigma_{0}$ présenté figure 10 souligne le caractère fortement non linéaire des relations traitées, il est déduit des formules générales établies au paragraphe (3) et de la loi de changement d'état. Plus particulièrement sur les segments $\left[10 \tilde{p}_{1}-\tilde{p}_{1}\right]$ et $\left[\tilde{p}_{1}-0\right]$ on a respectivement :

$$
\begin{aligned}
& \frac{\sigma}{\sigma_{0}}=\frac{\tilde{\sigma}}{\tilde{\sigma}_{0}}=\frac{f_{0}}{f} \frac{G_{11}^{3}}{G_{1}^{2}} \bar{A}_{1}^{3}, \\
& \frac{\sigma}{\sigma_{0}}=\frac{\tilde{\sigma}}{\tilde{\sigma}_{0}}=\frac{f_{0}}{f} \bar{A}_{1}^{3} .
\end{aligned}
$$

Concernant l'ellipticité $k_{0}=1$, la figure 11, qui compare les résultats de FLAHERTY et coll. [3] avec la courbe $\sigma / \sigma_{0}$, montre que nos résultats sont conformes à ces données de la littérature. Il est à souligner que pour $\tilde{p}<\tilde{p}_{1}$ la correspondance est rigoureuse puisqu'induite par la loi de similitude. D'autre part, les résultats d'un calcul numérique direct, figure 12, semblable à celui décrit plus haut, limité à sept sections pour les ellipticités 2 et 5 , confortent l'approche pragmatique développée dans le cadre de cet article.

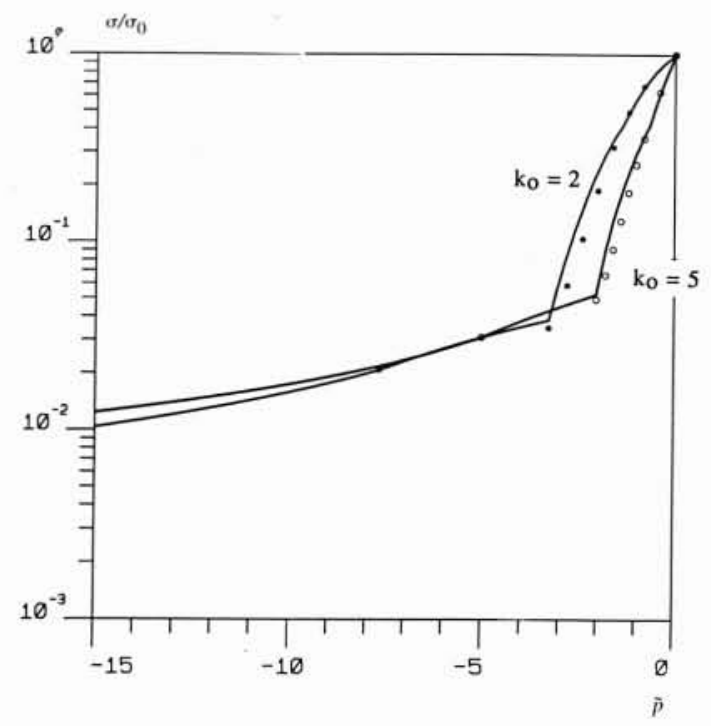

12. Rapport des conductances $\sigma$ par la conductance $\sigma_{0}$ dans des conduites cylindriques issues de la déformation d'un tube initialement elliptique $\left(k_{0}=2\right.$ et 5$)$ en fonction de $\tilde{p}$. Comparaison avec des valeurs calculées numériquement (symboles en forme d'étoile et de cercle).

The $\sigma$ to $\sigma_{0}$ conductivities ratio of cylindrical buckled tubes, that results from the collapse of an initially elliptical tube, $\left(k_{0}=2\right.$ et 5$)$ against $\tilde{p}$. Comparison with values numerically computed (star and circle symbols). 


\begin{tabular}{|c|c|c|c|}
\hline $\begin{array}{c}\text { Valeurs } \\
\text { normalisées A/A。 }\end{array}$ & 0.945 & 0.664 & 0.317. \\
\hline $\begin{array}{c}\text { Valeurs } \\
\text { expérimentales } \\
\text { de f }\end{array}$ & 68.9 & 73.1 & 50.0 \\
\hline $\begin{array}{c}\text { Valeurs } \\
\text { théoriques de f }\end{array}$ & 67.4 & 65.1 & 42.6 \\
\hline
\end{tabular}

Comparaison entre les valeurs théoriques et les valeurs expérimentales obtenues lorsque $k_{0}$ vaut 1,6 ./The theoretical values of the hydraulic shape factor compared with the experimental values obtained when $k_{0}=1.6$.

Les résultats des expériences de NAHMIAS [7], reprises en partie au laboratoire sur les mêmes canaux, soit pour $k_{0}=1,6$, montrent, table ci-dessus, que le modèle approche correctement les valeurs de $f$ dans l'intervalle $\left[\bar{A}_{p}-1\right]$ mais les sous-estime dans l'intervalle $\left[\bar{A}_{1}-\bar{A}_{p}\right]$. Néanmoins, ils renforcent l'argument d'une variation importante de $f(\bar{A})$ pour des valeurs de $\bar{A}$ légèrement supérieures à $\bar{A}_{c}$ et indiquent clairement que les valeurs maximales sont atteintes pour des aires normalisées inférieures à 0,78 (environ 0,6 pour $\left.k_{0}=1,6\right)$.

\section{Conclusion}

Bien qu'approchées, les fonctions $f(\bar{A}) / f_{0}$ proposées permettent de déterminer la loi cie perte de charge régulière de l'écoulement laminaire dans une conduite cylindrique de forme complexe qui résulte de l'écrasement d'un tube initialement elliptique. Nos résultats montrent que l'ellipticité initiale $k_{0}$ joue un rôle important dans la variation du coefficient de forme ou de la conductance, notamment pour $\bar{A}_{1} \leqslant \bar{A} \leqslant \bar{A}_{p}$. Nous montrons également que le coefficient de forme $f$ atteint une valeur minimale dans la section $\tilde{S}_{1}$. Cette valeur est inhabituellement basse: 36,5 et 33,2 aux bornes de l'intervalle $k_{0}=[1-10]$.

Par ailleurs ce calcul rend possible l'étude unidimensionnelle de l'écoulement permanent dans une conduite élastique collabable.

\section{Remerciements}

Nous tenons à remercier 1'Unité INSERM U2 et 1'Université Paris 12-Val de Marne pour le support financier de cette étude ainsi que le Laboratoire de Mécanique Expérimentale des Fluides de l'Université Pierre et Marie Curie pour le prêt des canaux d'expérimentation.

\section{Bibliographie}

[1] Bonis M., Ribreau C., Verchery G. (1981). - Etude expérimentale et théorique de l'aplatissement d'un tube élastique en dépression. $J$. de Méc. Appl., 5, 123-144.

[2] ConRad W.A. (1979). - A new model of the vocal cords based on a collapsible tube analogy. Medical Research Engineering, 13, $\mathrm{N}^{*} 2,7-10$.

[3] Flaherty J.E., Keller J.B., Rubinow S.I. (1972). Post buckling behaviour of elastic tubes and rings with opposite sides in contact. SIAM J. Appl. Math., 23, 446455.

[4] GRIFFiths D.J. (1971). - Hydrodynamics of male micturition-I. Theory of steady flow through elastic-walled tubes. Med. \& Biol. Engng., 9, Apr., 581-588.

[5] Hill R., Power G. (1956). - Extremum principles for slow viscous flow and the approximate calculation of drag. J. Mech. \& Appl. Math., 9, 313-319.

[6] KАмм R.D. (1982). - Bioengineering studies of periodic external compression as prophylaxis against deep vein thrombosis. Part I: numerical studies. ASME. J. Biomech. Engng., 104, May, 87-95.

[7] Nahmias J. (1980). - Perte de charge dans les tuyaux collabables. Thèse de $3^{\mathrm{e}}$ cycle. Université Paris 6.
[8] Pedley T.J. (1987). - How giraffes prevent oedema. Nature, 329, Sept., 13-14.

[9] Ribreau C. (1989). - Hémodynamique veineuse, équations de base. Journal des Maladies Vasculaires, 14, 287. 293.

[10] Ribreau C. (1991). - Sur la loi d'état, la loi de perte de charge, et la nature de l'écoulement permanent en conduite collabable inclinée. Thèse d'Etat, Université Paris 12-Val de Marne.

[11] Rodbard S. (1966). - A Hydrodynamic Mechanisin for Autoregulation of Flow. Cardiologia, 48, 6, 532-535.

[12] ThIRIET M., Bonis M. (1983). - Experiments on flow limitation during forced expiration in a monoalveolar lung model. Med. \& Biol. Engng. \& Comput., 21, Nov., 681-687.

[13] Vischer D., Peter P. (1985). - Le manchon souple d'étranglement, organe autorégulateur de répartition des débits constants dans les canaux d'irrigation. $L a$ Houille Blanche, 2, 123-132.

[14] WALkeR J. (1987). - Expériences d'amateur: Arrosez rapidement votre jardin... en bouchant le tuyau. Pour la Science, Sept., 94-98.

[15] ZARLing J.P. (1976). - An analysis of laminar flow and pressure drop in complex shaped ducts. J. Fluids Engng., 702-706. 